\title{
Afatinib, an EGFR inhibitor, decreases EMT and tumorigenesis of Huh-7 cells by regulating the ERK-VEGF/MMP9 signaling pathway
}

\author{
YAFEI CHEN, XIN CHEN, XIAOJUN DING and YINGWEI WANG \\ Department of Clinical Laboratory, Tiantai People's Hospital, Taizhou, Zhejiang 317200, P.R. China
}

Received July 2, 2018; Accepted April 24, 2019

DOI: $10.3892 / \mathrm{mmr} .2019 .10562$

\begin{abstract}
Transcatheter arterial embolization (TAE) therapy has been used in the treatment of inoperable hepatocellular carcinoma (HCC). However, tumor recurrence and metastasis are common in patients after TAE, and these processes may be caused by circulating tumor cells (CTCs). Epithelial-mesenchymal transition (EMT) serves important roles in CTCs, and abnormal expression and activation of epidermal growth factor receptor (EGFR) is common in cancer cells. Afatinib is an EGFR-tyrosine kinase inhibitor (TKI). The present study aimed to investigate the effects of afatinib on EMT and tumorigenesis in HCC cells. Western blot analysis suggested that afatinib was able to effectively suppress overactivation of EGFR. Moreover, the expression levels of EMT- and metastasis-associated genes were found to be modulated by afatinib through EGFR inhibition. In addition, Cell Counting Kit-8 and Transwell assays suggested that the viability, migration and invasion of HCC cells were inhibited by afatinib through EGFR inhibition. Furthermore, the activity of the ERK signaling pathway and the expression levels of vascular endothelial growth factor (VEGF) and matrix metalloproteinase 9 (MMP9) were decreased following treatment with afatinib in vitro. Collectively, the present results suggested that the inhibitory effects of afatinib on EMT and tumorigenesis may be associated with the ERK-VEGF/MMP9 signaling pathway. The present study provides new insights into understanding the mechanism underlying HCC and may facilitate the development of novel therapeutic strategies to treat $\mathrm{HCC}$ recurrence.
\end{abstract}

Correspondence to: Dr Yingwei Wang, Department of Clinical Laboratory, Tiantai People's Hospital, 335 Labor Road, Taizhou, Zhejiang 317200, P.R. China

E-mail: yingweiw_wyw@163.com

Key words: afatinib, epidermal growth factor, ERK, vascular endothelial growth factor/matrix metalloproteinase 9, hepatocellular carcinoma

\section{Introduction}

Primary hepatocellular carcinoma (HCC) is a malignancy with high mortality rate, and it is common in developing countries (1). HCC is a leading cause of tumor-associated mortality in China, partly due to the high occurrence rate of chronic hepatitis B virus infection (2). Transcatheter arterial embolization (TAE) has been widely used for the treatment of inoperable hepatocellular carcinoma, and it has been shown to decrease the tumor volume with promising effects (3); however, tumor recurrence and metastasis are common after TAE $(4,5)$, and these processes may be caused by circulating tumor cells (CTCs). CTCs have the ability to invade distant tissues and to survive in various microenvironmental contexts (6). The expression of epidermal growth factor receptor (EGFR), a transmembrane tyrosine kinase receptor (7), has been identified to have an important role in CTCs (6). EGFR is dysregulated in many types of cancer, including HCC (8). Although EGFR signaling was identified to be critical for HCC growth and metastasis, and a previous study identified that treatment with the EGFR inhibitor cetuximab could reduce HCC growth following TAE, the effects of other EGFR inhibitors, such as afatinib, and their possible regulatory mechanisms in $\mathrm{HCC}$ cells following TAE remain unknown $(9,10)$. Afatinib, an EGFR-tyrosine kinase inhibitor (TKI), was previously identified to repress cancer cell growth by mitigating the activation of EGFR in tumor cells (11). A previous study reported that epithelial-mesenchymal transition (EMT) has a critical role in the shedding of CTCs from primary tumors into blood vessels, and EMT is an essential process in embryonic development and cancer progression (12). EMT is characterized by a loss of cell polarity, cell-cell adhesion, and increased migratory and invasive abilities, which facilitate the metastasis of cancer cells $(13,14)$. E-cadherin and Vimentin are involved in the EMT process (15). In addition, metastasis-associated gene 1 (MTA1) $(16,17)$ and T lymphoma invasion and metastasis inducing factor 1 (TIAM1) are responsible for cell migration and adhesion in tumorigenesis (18-20). Therefore, afatinib may be involved in preventing EMT in HCC following TAE.

Vascular endothelial growth factor (VEGF) is a potent inducer of angiogenesis and was identified to be associated with tumor angiogenesis (21). Matrix metalloproteinase (MMPs) play critical roles in proteolytic degradation and in altering cell adhesion, cell migration and EMT during cancer-associated 
angiogenesis (22-24). ERK is a member of the mitogen-activated protein kinase (MAPK) signaling cascade (25), and was identified to be involved in multiple biological processes regulated by phosphorylation cascades, including gene expression, cell survival and cell migration (26-28). Therefore, the present study aimed to investigate the effects of afatinib on HCC after TAE and the potential mechanisms underlying its function. The present results suggested that afatinib was able to effectively suppress the overactivation of EGFR. Moreover, the expression levels of genes involved in proliferation, migration, invasion and EMT were decreased following treatment with afatinib through EGFR inhibition in HCC cells, and these effects may be associated with the ERK-VEGF/MMP signaling pathway. The present results may provide new insights into the mechanisms underlying the prevention of HCC recurrence after TAE.

\section{Materials and methods}

Tissue specimens. In total, 50 patients with HCC underwent TAE intervention therapy in Tiantai County People's Hospital between June 2014 and April 2016. The inclusion criteria were the following: i) All patients with HCC were diagnosed by histological analysis; ii) all patients underwent curative surgery with no presurgical treatment resulting in tissue necrosis; iii) the patients did not receive radiotherapy or chemotherapy before surgical intervention; and iv) no patient had concurrent presence of another liver carcinoma. Signed written informed consent was obtained from 42 patients for the use of their clinical tissues. Eight patients did not provide written informed consent and were thus excluded from the present study. The adjacent normal tissues were collected at a distance of $5 \mathrm{~cm}$ from the tumor margin. The median age was 50 years (range, 30-89 years). The tumor length was between 0.5 and $8.5 \mathrm{~cm}$ (median, $5 \mathrm{~cm}$ ). The protocols were performed according to the Declaration of Helsinki. Paired tissues were divided into two groups. Sections were stored in $4 \%$ formaldehyde at $-80^{\circ} \mathrm{C}$ for routine pathological diagnosis, whereas the other part was frozen by immersion into liquid nitrogen and stored at $-80^{\circ} \mathrm{C}$. Reverse transcription-quantitative PCR (RT-qPCR) and western blot analysis were performed to examine the tissues. The association between the expression level of EGFR and clinicopathological features is presented in Table I. The present study was approved by The Ethics Committee of Tiantai County People's Hospital.

Cell culture and treatment. Huh-7 cells were purchased from Thermo Fisher Scientific, Inc. The cells were maintained at $37^{\circ} \mathrm{C}$ in an incubator with $5 \% \mathrm{CO}_{2}$ and cultured in DMEM (Gibco; Thermo Fisher Scientific, Inc.) supplemented with 10\% FBS (Gibco; Thermo Fisher Scientific, Inc.), streptomycin and penicillin (CoWin Biosciences Co., Ltd.). Cells were plated in 6-well plates at a density of $1 \times 10^{5}$ cells/well and were starved overnight before treatment. On the following day, the cells were treated with $25 \mathrm{ng} / \mathrm{ml}$ recombinant human EGF (PeproTech, Inc.) for $6 \mathrm{~h}$ to mimic the overactivation of EGFR in HCC after TAE.

Cell transfection. Subsequently, the cells were transfected with small interfering RNAs (siRNAs). EGFR siRNA (si-EGFR) and scrambled siRNA (si-CTR) were purchased from Shanghai GenePharma Co., Ltd. In total, $0.25 \mu \mathrm{g}$ siRNA was transfected into Huh-7 cells, using Lipofectamine 3000 (Invitrogen; Thermo Fisher Scientific, Inc.) as transfection reagent. $24 \mathrm{~h}$ after transfection, cells were used for following detection. Subsequently, $10 \mathrm{nmol}$ afatinib (Selleck Chemicals) was added to $100 \mu \mathrm{l}$ cell culture medium for $18 \mathrm{~h}$ at $37^{\circ} \mathrm{C}$. Treatment with DMSO $(0.1 \%)$ was used as the control.

Assessment of cell viability. After $18 \mathrm{~h}$ of treatment with afatinib, the cells were plated at a density of $1 \times 10^{3}$ cells/well in 96-well plates. Then, cell viability was detected using a Cell Cycle Kit-8 (CCK-8) according to the manufacturer's protocol (Beyotime Institute of Biotechnology). The CCK-8 solution was added and incubated at $37^{\circ} \mathrm{C}$. After $4 \mathrm{~h}$, the medium was removed and a microplate reader (Bio-Rad Laboratories, Inc.) was used for determining absorbance values at $450 \mathrm{~nm}$.

Detection of migratory and invasive abilities of Huh-7 cells. Matrigel-coated Transwell inserts (Corning, Inc.) were used to measure the migratory and invasive abilities of cancer cells, as previously described (29). For the migration assay, the inserts were not coated with Matrigel. Cells were plated at a concentration of $2 \times 10^{5}$ cells $/ \mathrm{ml}$ in the upper chamber and incubated for $24 \mathrm{~h}$. Crystal violet $(0.1 \%)$ was used for staining at $37^{\circ} \mathrm{C}$ for $20 \mathrm{~min}$. The migrated or invaded cells were counted for 200 fold using a light microscope (Nikon Corporation; magnification, x200), and the averages were calculated.

$R T-q P C R$. The relative gene expression data were analyzed by RT-qPCR. Total RNA was extracted using RNeasy kit (Qiagen $\mathrm{GmbH})$. Subsequently, $1 \mu \mathrm{g}$ RNA was reversely transcribed to cDNA using High-capacity cDNA Reverse Transcription Kit (Applied Biosystems; Thermo Fisher Scientific, Inc.), according to the manufacturer's protocol. The qPCR was performed using the ChamQ SYBR qPCR master mix (Vazyme) on a Bio-Rad CFX96 system. The thermocycling conditions were the following: Initial denaturation at $95^{\circ} \mathrm{C}$ for $15 \mathrm{sec}$, followed by 40 cycles of $95^{\circ} \mathrm{C}$ for $25 \mathrm{sec}, 55^{\circ} \mathrm{C}$ for $25 \mathrm{sec}$ and $72^{\circ} \mathrm{C}$ for $30 \mathrm{sec}$. RT-qPCR data were quantified using the $2^{-\Delta \Delta \mathrm{Cq}}$ method (30). GAPDH was used as internal control gene. The sequences of the primers used in the present study are listed in Table II.

Western blotting. Total protein was extracted using RIPA buffer (Boster Biological Technology) and $1 \mathrm{mmol} / 1 \mathrm{PMSF}$ and separated by SDS-PAGE on $12 \%$ gels. Next, the proteins were transferred to PVDF membranes (EMD Millipore). Subsequently, 2\% BSA (Beijing Solarbio Science \& Technology Co., Ltd.) was added to block nonspecific binding. The PVDF membranes were incubated overnight at $4^{\circ} \mathrm{C}$ with primary antibodies (all from Cell Signaling Technology, Inc.) against phosphorylated (p-)EGFR (cat. no. 4407, 1:1,000), EGFR (cat. no. 3197; 1,000), MMP9 (cat. no. 3852; 1,000), p-ERK (cat. no. 9101; 1,000), ERK (cat. no. 9102; 1,000), VEGF (cat. no. $2463 ; 1,000)$, E-cadherin (cat. no. 3195; 1,000), Vimentin (cat. no. 5741; 1,000), TIAM1 (cat. no. 63647; 1,000), MTA1 (cat. no. 5646; 1,000) and GAPDH (cat. no. 2118; 1:2,000). After being washed with PBS, the membranes were incubated with a horseradish peroxidase-labeled secondary antibody (cat. no. 7074; 1:5,000; Cell Signaling Technology, Inc.). The bands were visualized using an ECL kit (Pierce; Thermo 
Table I. Association between EGFR expression and various clinicopathological features.

Expression of EGFR

\begin{tabular}{|c|c|c|c|c|}
\hline & & & & \\
\hline Clinicopathological features & $\mathrm{n}$ & Positive, $\mathrm{n}(\%)$ & Negative, n (\%) & P-value \\
\hline Sex & & & & 0.798 \\
\hline Male & 32 & $11(34.4)$ & $21(65.6)$ & \\
\hline Female & 10 & $3(30.0)$ & $7(70.0)$ & \\
\hline Age, years & & & & 0.172 \\
\hline$\leq 50$ & 15 & $7(46.7)$ & $8(53.3)$ & \\
\hline$>50$ & 27 & $7(25.9)$ & $20(74.1)$ & \\
\hline Histology differentiation & & & & 0.127 \\
\hline High & 8 & $5(62.5)$ & $3(37.5)$ & \\
\hline Moderate & 19 & $7(36.8)$ & $12(63.2)$ & \\
\hline Low & 15 & $2(20.0)$ & $13(80.0)$ & \\
\hline Liver cirrhosis & & & & 0.35 \\
\hline Yes & 36 & $11(30.6)$ & $25(69.4)$ & \\
\hline No & 6 & $3(50.0)$ & $3(50.0)$ & \\
\hline$\alpha$-fetoprotein & & & & 0.469 \\
\hline$\leq 400$ & 30 & $11(36.7)$ & $19(63.3)$ & \\
\hline$>400$ & 12 & $3(25.0)$ & $9(75.0)$ & \\
\hline Tumor diameter, $\mathrm{cm}$ & & & & 0.116 \\
\hline$\leq 5$ & 26 & $11(42.3)$ & 15 (57.7) & \\
\hline$>5$ & 16 & $3(18.8)$ & $13(81.3)$ & \\
\hline TNM stage & & & & 0.075 \\
\hline $\mathrm{I} / \mathrm{II}$ & 25 & $11(44.0)$ & $14(56.0)$ & \\
\hline III/IV & 17 & $3(17.6)$ & $14(82.4)$ & \\
\hline Intravascular tumor thrombus & & & & $0.028^{\mathrm{a}}$ \\
\hline Yes & 19 & $3(15.8)$ & $16(84.2)$ & \\
\hline No & 23 & $11(47.8)$ & $12(52.2)$ & \\
\hline Portal vein tumor thrombus & & & & 0.736 \\
\hline Yes & 5 & $2(40.0)$ & $3(60.0)$ & \\
\hline No & 37 & $12(32.4)$ & $25(67.6)$ & \\
\hline Involving the liver capsule & & & & $0.005^{\mathrm{a}}$ \\
\hline Yes & 32 & $7(21.9)$ & $25(78.1)$ & \\
\hline No & 10 & $7(70.0)$ & $3(30.0)$ & \\
\hline
\end{tabular}

${ }^{a} \mathrm{P}<0.05$. EGFR, epidermal growth factor receptor; TNM, tumor, node and metastasis.

Fisher Scientific, Inc.). Digital images of immunoreactive bands were analyzed using the Bio-Rad ChemiDoc XRS+ System with Image Lab Software (version 1708265; Bio-Rad Laboratories, Inc.).

Statistical analysis. SPSS 22.0 (IBM Corp.) and GraphPad Prism 6 software (GraphPad Software, Inc.) were used for statistical analysis. Data are presented as the mean \pm SD. The $\chi^{2}$ test was used to analyze the association between the expression level of EGFR and various clinical features or between the expression levels of EGFR and E-cadherin. One-way ANOVA followed by Dunnett's post-hoc test was used to compare multiple groups. $\mathrm{P}<0.05$ was considered to indicate a statistically significant difference.

\section{Results}

Expression level of EGFR in HCC after TAE. The mRNA and protein expression level of EGFR in HCC tissues was investigated using RT-qPCR and western blot analysis, respectively. The results suggested that EGFR was upregulated in HCC tissues and increased following TAE compared with the control group (Fig. 1). The association between the expression level of EGFR and various clinicopathological features was also examined (Table I). The expression level of EGFR was significantly associated with intravascular tumors and the retraction/lesion of the liver capsule, but not with gender, age and tumor diameter. In addition, the expression level of E-cadherin was significantly reduced in HCC tissues, in 
Table II. Reverse transcription-quantitative PCR primers.

\begin{tabular}{ll} 
Gene symbol & \multicolumn{1}{c}{ Primer sequences $\left(5^{\prime}-3^{\prime}\right)$} \\
\hline EGFR & F: GCGCTACCTTGTCATTCAGG \\
& R: TATCAATGCAAGCCACGGTG \\
E-cadherin & F: TCACATCCTACACTGCCCAG \\
& R: AGTGTCCCTGTTCCAGTAGC \\
MMP9 & F: GCGTCTTCCCCTTCACTTTC \\
& R: ATAGGGTACATGAGCGCCTC \\
VEGF & F: TTGCTGTGCTTTGGGGATTC \\
& R: CTGTCATGGGCTGCTTCTTC \\
Vimentin & F: GAGAGGAAGCCGAAAACACC \\
& R: TTCCTGAATCTGAGCCTGCA \\
MTA1 & F: ACAGACAAGCAGATCGACCA \\
& R: GGCCTTGGAGATGTCGTAGA \\
TIAM1 & F: ACTGTCTCTCTGAAGGTGCC \\
& R: GGTGAGTAGCTGGAGTTGGT \\
GAPDH & F: CACAGTCCATGCCATCACTG \\
& R: ATCTCGCTCCTGGAAGATGG
\end{tabular}

EGFR, epidermal growth factor receptor; F, forward; R, reverse; MMP, matrix metalloproteinase; VEGF, vascular endothelial growth factor; MTA1, metastasis associated 1; TIAM1, T cell lymphoma invasion and metastasis 1 .

particular after TAE (Fig. 1). Although the expression levels of EGFR and E-cadherin were not significantly associated with each other, 18 patients $(66.7 \%)$ exhibited positive expression of EGFR and negative expression of E-cadherin (Table III). Sample exhibiting higher levels than the control group were considered positive, whereas samples with lower levels were considered negative.

Afatinib modulates the expression levels of EMT- and metastasis-associated genes in HCC cells. EMT is involved in the migration of tumor cells, thus increasing the tumor metastatic ability (31). RT-qPCR and western blot analysis showed that knockdown of EGFR expression by siRNA decreased the protein expression level ratio of $\mathrm{p}$-EGFR/EGFR. Moreover, afatinib could effectively suppress the phosphorylation of EGFR; whereas the knockdown of EGFR expression by siRNA decreased the ratio of $p-E G F R / E G F R$. Moreover, afatinib could effectively decrease the ratio of $\mathrm{p}$-EGFR/EGFR (Fig. 2A-C). Additionally, the mRNA and protein expression levels of EMT- and metastasis-associated genes were examined by RT-qPCR and western blot, respectively. The expression levels of E-cadherin and TIAM1, which are negative regulators of EMT $(32,33)$, were increased following EGFR knockdown or afatinib-mediated inhibition of EGFR. However, the expression levels of Vimentin and MTA1, which are positive regulators of EMT $(34,35)$, were decreased in EGFR knockdown group or the afatinib group (Fig. 2D-F).

Afatinib inhibits viability, migration and invasion of $\mathrm{HCC}$ cells. The effects of afatinib on HCC tumorigenesis were investigated in vitro. The CCK-8 results suggested that cell viability
Table III. Association between the expression levels of EGFR and E-cadherin.

\begin{tabular}{lrrrrr}
\hline & \multicolumn{2}{c}{$\begin{array}{c}\text { E-cadherin } \\
\text { expression }\end{array}$} & & \\
\cline { 2 - 4 } EGFR expression & $-(\%)$ & $+(\%)$ & n & P-value \\
\cline { 2 - 4 }+ & $7(46.7)$ & $8(53.3)$ & 15 & 0.206 \\
\hline & $18(66.7)$ & $9(33.3)$ & 27 & \\
\hline
\end{tabular}

EGFR, epidermal growth factor receptor.

was reduced (Fig. 3A) in the EGFR-TKI group. Moreover, the Transwell assays suggested that the migratory and invasive abilities of HCC cells were significantly decreased following treatment with afatinib (Fig. 3B-E). Collectively, the present results suggested that afatinib could inhibit the viability, migration and invasion of HCC cells through EGFR inhibition.

Activity of the ERK-VEGF/MMP9 signaling pathway is decreased by afatinib through EGFR inhibition. To investigate the molecular mechanisms underlying afatinib function, the activity of ERK and the expression levels of VEGF and MMP9 were investigated following treatment with afatinib. RT-qPCR and western blot analysis results suggested that the expression levels of VEGF and MMP9 were decreased by afatinib through EGFR inhibition (Fig. 4A and B). Moreover, the phosphorylation level of ERK was significantly decreased after treatment with afatinib through EGFR inhibition, and the ratio of p-ERK/ERK was significantly decreased after treatment with afatinib through EGFR inhibition (Fig. 4C and D). The present results suggested that afatinib decreased the activity of the ERK-VEGF/MMP9 signaling pathway in vitro.

\section{Discussion}

HCC is a type of malignant cancer with high incidence rates worldwide (1). TAE is an effective palliative treatment for inoperable HCC. Nevertheless, a high incidence of HCC recurrence and metastasis was reported after TAE (5). Multiple intracellular signals are altered in HCC cells following TAE, leading to an increased number of CTCs, thus promoting metastasis and tumor recurrence $(4,5)$. As one of the most widely investigated receptor tyrosine kinase families, EGFRs serve important roles in signal transduction and oncogenesis (36). Previous studies reported that EGFR signaling is critical for HCC growth, metastasis and invasion $(37,38)$. Additionally, dysregulation of EGFR is common in certain types of tumors (39). Accumulating evidence suggested that EGFR is overactivated or mutated in HCC; Ikeda et al (40) performed next-generation sequencing to investigate circulating tumor DNA, and found that $14 \%$ of patients with $\mathrm{HCC}$ presents an increased number of copies of EGFR. EGFR was activated in HCC cells (41). In addition, Panvichian et al (42) demonstrated that EGFR overexpression and mutations in the EGFR gene are present in HCC. EMT contributes to the growth and metastasis of cancer cells in the 

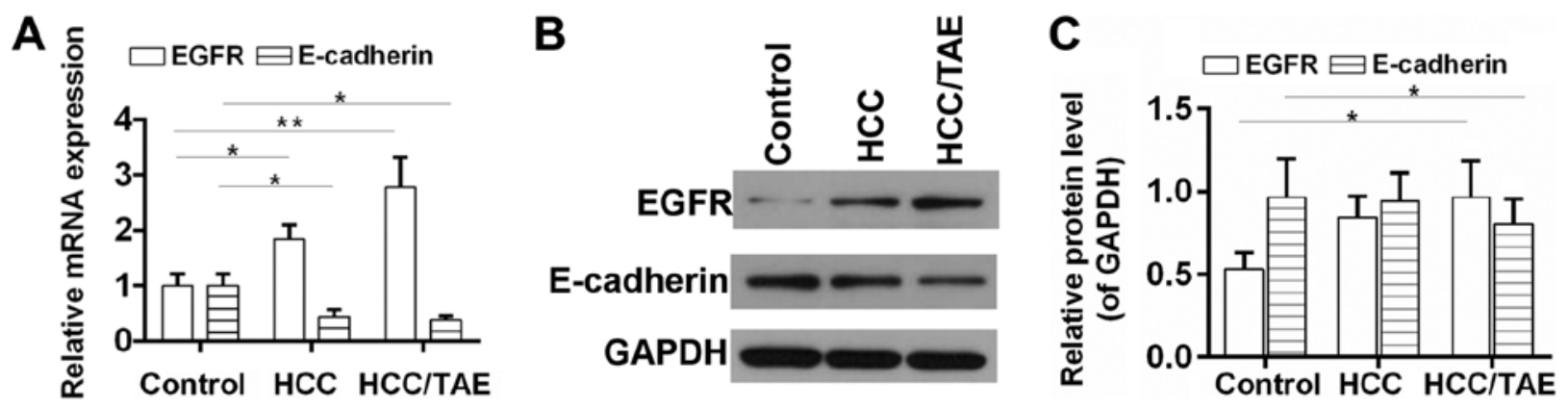

Figure 1. Detection of the expression levels of EGFR and E-cadherin in HCC tumor samples. (A) Reverse transcription-quantitative PCR and (B) western blot analysis of EGFR and E-cadherin. (C) Densitometric analysis of the protein expression levels of EGFR and E-cadherin. $n=42 .{ }^{*} \mathrm{P}<0.05,{ }^{* *} \mathrm{P}<0.01$. EGFR, epidermal growth factor receptor; TAE, transcatheter arterial embolization; HCC, hepatocellular carcinoma.

A

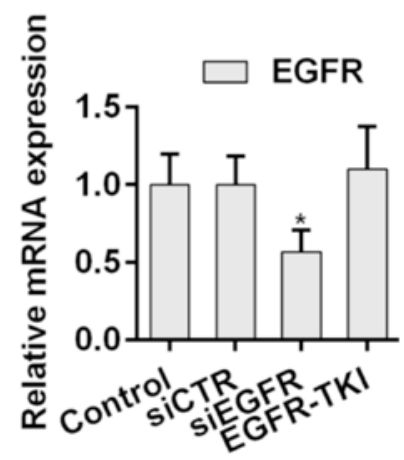

D

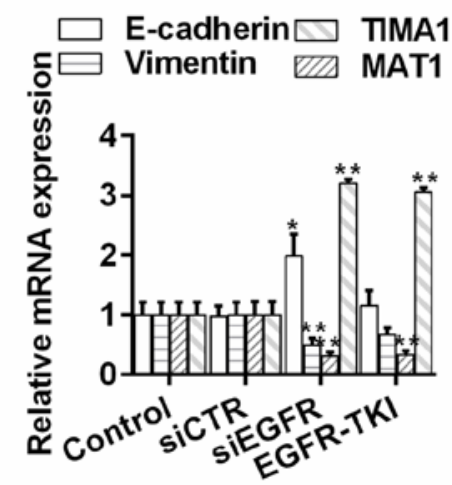

B

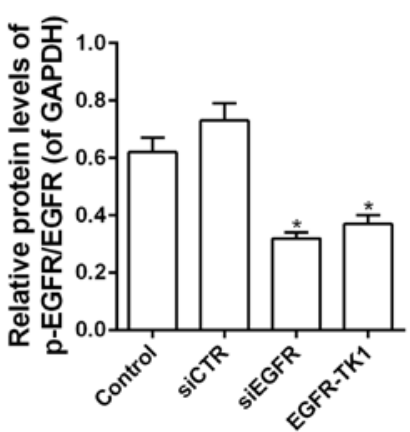

E

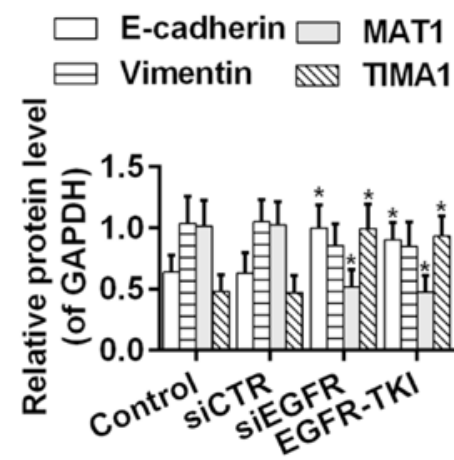

C

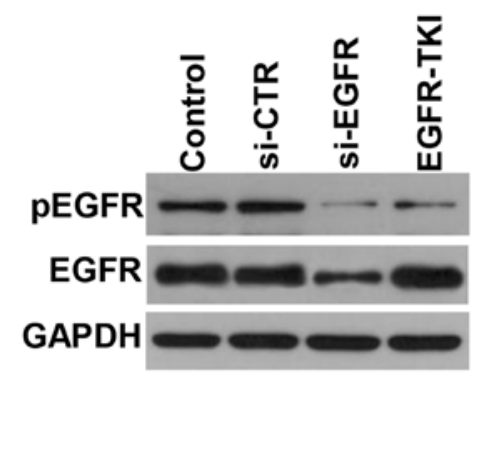

$\mathbf{F}$

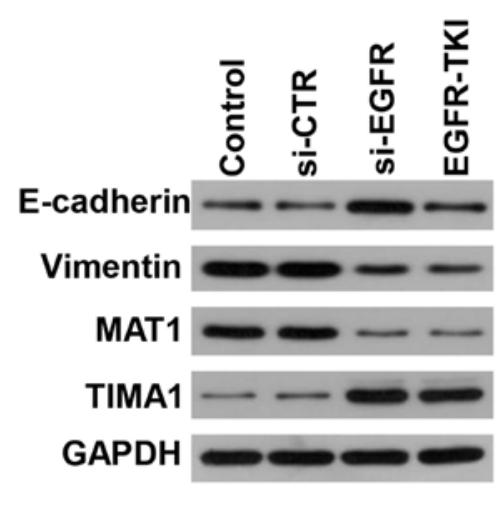

Figure 2. Detection of the expression levels of EGFR, E-cadherin, Vimentin, MTA1 and TIAM1 in HCC tumor samples. (A) mRNA and (B) protein expression level of EGFR. (C) Western blot analysis of EGFR and p-EGFR. (D) mRNA and (E) protein expression levels of E-cadherin, Vimentin, MTA1 and TIAM1. (F) Western blot analysis of E-cadherin, Vimentin, MTA1 and TIAM1. $\mathrm{n}=3$. ${ }^{*} \mathrm{P}<0.05,{ }^{* *} \mathrm{P}<0.01$ vs. corresponding control. EGFR, epidermal growth factor receptor; MTA1, metastasis associated 1; TIAM1, T cell lymphoma invasion and metastasis 1; si-CTR, scrambled siRNA; si-EGFR, siRNA targeting EGFR; siRNA, small interference RNA; p-, phosphorylated; EGFR-TKI, afatinib treatment; TKI, tyrosine-kinase inhibitor.

liver (31). VEGF has an important role in the proliferation and differentiation of endothelial cells (21). In addition, MMPs serve key roles in the degradation of basement membrane collagen and extracellular matrix (43). Interestingly, afatinib, a TKI able to suppress the activity of EGFR, can interact with the ATP-binding site of EGFR, blocking its enzymatic activity (44). In a previous study, EGFR ${ }^{\mathrm{WT}}$ and $\mathrm{EGFR}^{\mathrm{L} 858 \mathrm{R} / \mathrm{T} 790 \mathrm{M}}$ kinase inhibition assays identified that afatinib has significant inhibitory activities, and its $\mathrm{IC}_{50}$ values, tested in $\mathrm{A} 549$,
HepG2, MCF-7 and PC-3 cell lines, are in the nanomolar range (45). Moreover, afatinib was reported to have inhibitory effects on non-small-cell lung cancer (NSCLC) and has been approved for the therapy of metastatic NSCLC by the Food and Drug Administration $(45,46)$. Therefore, the present study aimed to investigate the effects of afatinib on $\mathrm{HCC}$ and its underlying molecular mechanism.

Fang et al (47), reported that possible mechanisms underlying the metastatic potential of HCC cells following TAE are 

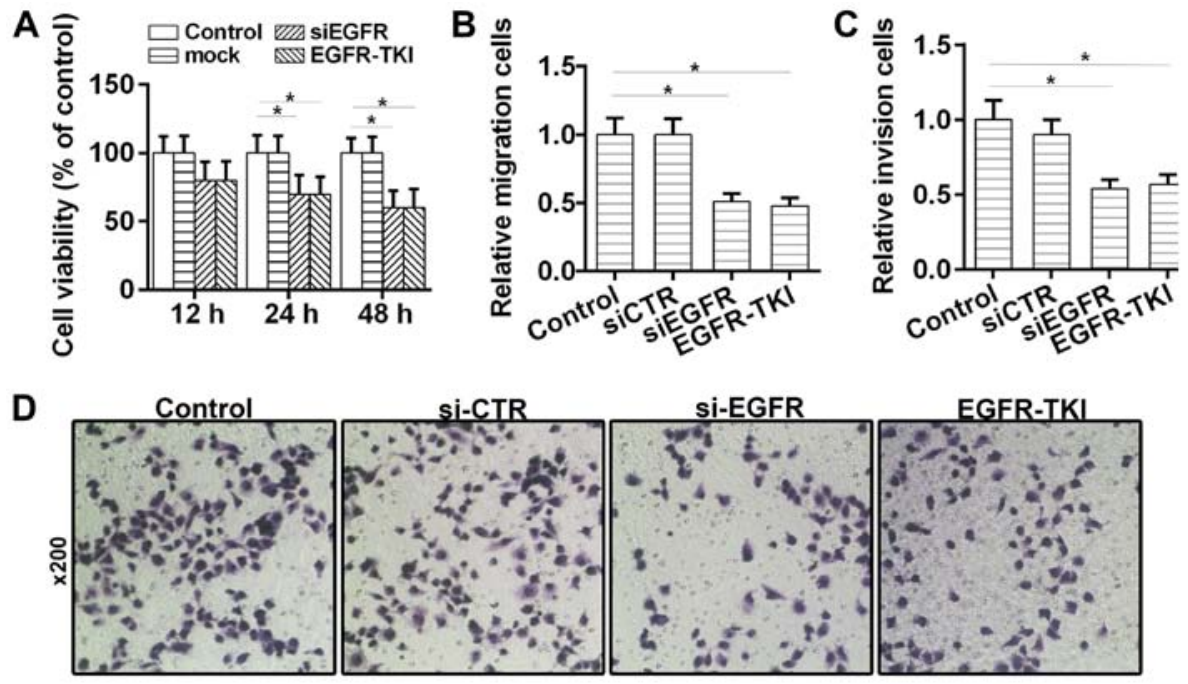

E

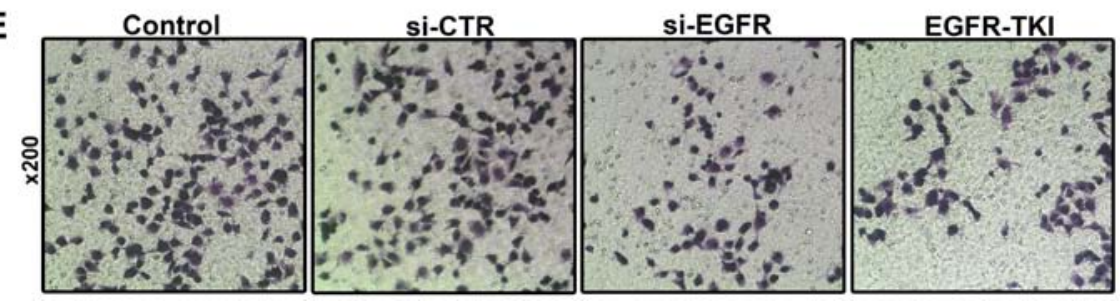

Figure 3. Effect of afatinib on the proliferation, migration and invasion of HCC cells. (A) Cell Cycle Kit-8 assay was performed to investigate cell viability. Relative quantification of (B) migration and (C) invasion of HCC cells. Transwell (D) migration and (E) invasion assay for the migration and invasion ability of HCC cells. $n=3$. "P<0.05. EGFR, epidermal growth factor receptor; si-CTR, scrambled siRNA; si-EGFR, siRNA targeting EGFR; siRNA, small interference RNA; EGFR-TKI, afatinib treatment; TKI, tyrosine-kinase inhibitor; HCC, hepatocellular carcinoma.

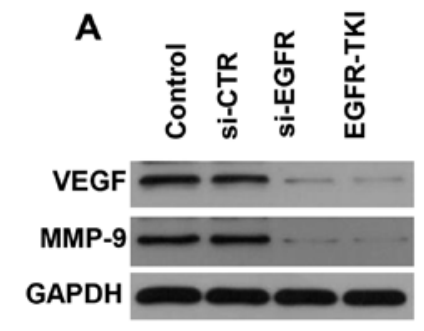

B

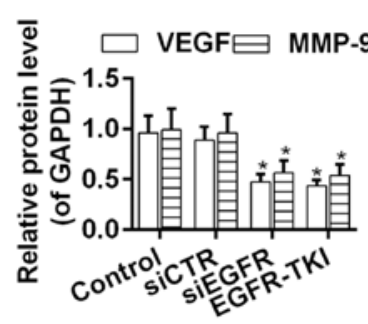

C

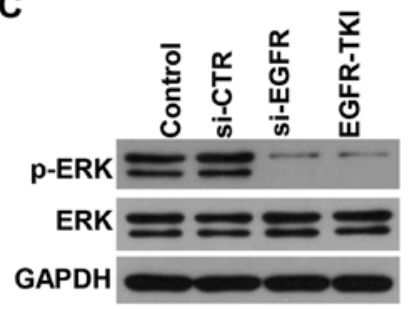

D

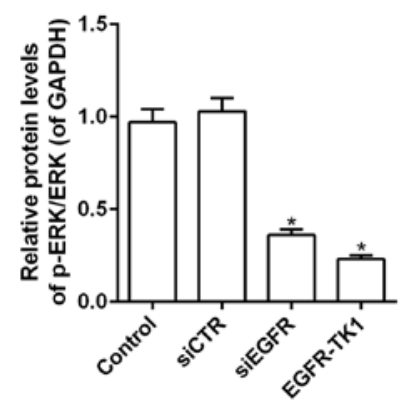

Figure 4. Effect of afatinib on the ERK-VEGF/MMP9 signaling pathway. (A) Western blot and (B) densitometry analysis of VEGF and MMP9 protein expression levels. (C) Western blot and (D) densitometry analysis of ERK and p-ERK levels. The ratio of $p$-ERK/total ERK was evaluated. $n=3$. " $P<0.05$ vs. corresponding control. EGFR, epidermal growth factor receptor; VEGF, vascular endothelial growth factor; MMP, matrix metalloproteinase; siCTR, scrambled siRNA; si-EGFR, siRNA targeting EGFR; siRNA, small interference RNA; EGFR-TKI, afatinib treatment; TKI, tyrosine-kinase inhibitor; p-, phosphorylated.

the acquisition of EMT features and the increased number of CTCs. The present study not only examined the expression levels of genes involved in the mechanism underlying HCC metastasis, but also suggested a possible EGFR-targeted therapeutic strategy.
E-cadherin is downregulated during cell migration and metastasis of tumor cells, which are associated with EMT (48-51). In the present study, the association between the expression levels of EGFR and E-cadherin was analyzed, and the present results suggested that the expression level of EGFR 
was not significantly associated with the expression level of E-cadherin; however, 18 patients $(66.7 \%)$ were EGFR-positive and E-cadherin-negative. A higher number of samples are required to confirm the association between the expression levels of these two genes. The present results suggested that EGFR could be used as a target for the prevention of EMT in HCC after TAE. In the present study, the effects of afatinib on the activity of EGFR signaling were investigated. Additionally, the expression levels of E-cadherin and Vimentin, two genes associated with EMT, increased and decreased following afatinib-mediated EGFR inhibition, respectively. Moreover, the expression levels of the metastasis-associated genes MTA1 and TIAM1 were decreased and increased in the EGFR-TKI group, respectively. Wang et al (52) suggested that silencing EGFR or inhibiting the phosphorylation of molecules downstream of the EGFR pathway could inhibit EMT and metastasis in HCC. Deng et al (53) reported that reducing the activity of HER2, a member of the EGFR family, can decrease the promotion of EMT by increasing the expression level of MTA1 in HCC both in vitro and in vivo, suggesting that the EGFR-MTA-EMT1 pathway may be context-specific. In addition, the acquisition of EMT features was observed in lung cancer cell lines, and is associated with an increased resistance to afatinib (54). Nevertheless, EMT-associated resistance to afatinib in the treatment of $\mathrm{HCC}$ requires further validation in clinical studies. A previous study reported that the effects of combined treatment with bufalin and afatinib is associated with the inhibition of EMT in lung cancer cells (55). Therefore, combined treatments and conversion therapies may be used in the treatment of HCC. In addition, CCK-8 and Transwell assays were performed to investigate the effects of afatinib on tumorigenesis. The present results suggested that afatinib decreased the proliferation, migration and invasion of HCC cells through EGFR inhibition. The inhibitory effect of afatinib on the migration and metastasis of HCC cells was in accordance with a previous study describing the effects of afatinib on NSCLC (46). In the present study, the molecular mechanism of afatinib was investigated in HCC cells.

The MAPK/ERK pathway is involved in numerous biological events, including cancer progression $(26,28,56)$. In the present study, the protein expression level of p-ERK was identified to be reduced by afatinib in vitro. The present results suggested that afatinib may exert its inhibitory effect on tumorigenesis by inactivating the ERK signaling pathway. The present findings are in line with a previous study that showed induction of apoptosis in Eca109 cells following knockdown of ERK2 (8). Nevertheless, a previous study reported that the effect of the ERK signaling pathway depends on the cellular context and the crosstalk with other signaling pathways (46). In addition, VEGF and MMPs are responsible for tumor angiogenesis, growth and metastasis (57-59). Zhang et al (60) investigated the effects of metformin in combination with curcumin on the growth and progression of $\mathrm{HCC}$, and the downregulation of the expression levels of MMP2/9, VEGF and VEGF receptor-2 were identified to be associated with the suppression of EGFR signaling. This previous study is in line with the present study, which suggested that the expression levels of VEGF and MMP9 were decreased by afatinib through EGFR inhibition in HCC cells. Previous studies demonstrated that the VEGF signaling pathway promotes the activation of MMPs in endothelial cells $(57,58,61)$. However, the present study did not investigate whether VEGF regulated MMP9, which were previously shown to be associated (62). Additionally, previous studies showed that the MAPK/ERK pathway is downstream of the VEGF signaling pathway $(63,64)$; in contrast, a previous study showed that the upregulation of VEGF is dependent on ERK activation (65). Therefore, ERK may regulate VEGF signaling in a positive feedback mechanism. Therefore, examining the hierarchy between ERK and VEGF could improve the understanding of the molecular mechanism investigated in the present study. However, the present findings may increase the understanding of HCC, facilitating the development of novel treatments. The present study presented certain limitations, including the lack of clinical studies. In addition, to examine the effects of agonists of the ERK signaling pathway may validate the molecular mechanism identified in the present study.

Collectively, the present study identified that afatinib could not only effectively suppress the proliferation, migration and invasion of HCC cells, but also regulated the expression levels of EMT- and metastasis-associated genes through EGFR inhibition. Furthermore, the inhibitory effects of afatinib on tumorigenesis were identified to be associated with the ERK-VEGF/MMP9 signaling pathway. The present findings may facilitate the development of a novel therapeutic strategy for the recurrence of HCC.

\section{Acknowledgements}

Not applicable.

\section{Funding}

No funding was received.

\section{Availability of data and materials}

The datasets used and/or analyzed during the present study are available from the corresponding author on reasonable request.

\section{Authors' contributions}

YC conceived and designed the present study. XC and XD performed the experiments. YW analyzed, collected and interpreted the data.

\section{Ethics approval and consent to participate}

The present study was approved by The Ethics Committee of Tiantai County People's Hospital. Written informed consent was obtained from 42 patients for the use of their clinical tissues.

\section{Patient consent for publication}

Not applicable.

\section{Competing interests}

The authors declare that they have no competing interests. 


\section{References}

1. Burkhart RA, Ronnekleiv-Kelly SM and Pawlik TM: Personalized therapy in hepatocellular carcinoma: Molecular markers of prognosis and therapeutic response. Surg Oncol 26: 138-145, 2017.

2. Torre LA, Bray F, Siegel RL, Ferlay J, Lortet-Tieulent J and Jemal A: Global cancer statistics, 2012. CA Cancer J Clin 65: 87-108, 2015

3. Nagao E, Hirakawa M, Soeda H, Tsuruta S, Sakai H and Honda H: Transcatheter arterial embolization for chest wall metastasis of hepatocellular carcinoma. World J Radiol 5: 45-48, 2013.

4. Wang R, Zhao N, Li S, Fang JH, Chen MX, Yang J, Jia WH, Yuan Y and Zhuang SM: MicroRNA-195 suppresses angiogenesis and metastasis of hepatocellular carcinoma by inhibiting the expression of VEGF, VAV2, and CDC42. Hepatology 58: 642-653, 2013.

5. Germano D and Daniele B: TIE2-expressing monocytes as a diagnostic marker for hepatocellular carcinoma correlates with angiogenesis. Hepatobiliary Surg Nutr 3: 166-167, 2014.

6. Serrano MJ, Alvarez-Cubero MJ, De Miguel Pérez D, Rodríguez-Martínez A, Gonzalez-Herrera L, Robles-Fernandez I, Hernandez JE, Puche JLG and Lorente JA: Significance of EGFR expression in circulating tumor cells. Adv Exp Med Biol 994: 285-296, 2017.

7. Schlessinger J: Ligand-induced, receptor-mediated dimerization and activation of EGF receptor. Cell 110: 669-672, 2002.

8. Komposch K and Sibilia M: EGFR signaling in liver diseases. Int J Mol Sci 17: E30, 2015.

9. Zhong L, Liao D, Zhang M, Zeng C, Li X, Zhang R, Ma H and Kang T: YTHDF2 suppresses cell proliferation and growth via destabilizing the EGFR mRNA in hepatocellular carcinoma. Cancer Lett 442: 252-261, 2019.

10. Zhu L, Liu R, Zhang W, Qian S and Wang J: Application of EGFR inhibitor reduces circulating tumor cells during transcatheter arterial embolization. Clin Transl Oncol 20: 639-646, 2018.

11. Keating GM: Afatinib: A review in advanced non-small cell lung cancer. Target Oncol 11: 825-835, 2016.

12. Rhim AD, Mirek ET, Aiello NM, Maitra A, Bailey JM, McAllisterF, Reichert M, Beatty GL, Rustgi AK, Vonderheide RH, et al: EMT and dissemination precede pancreatic tumor formation. Cell 148: 349-361, 2012.

13. Li JY, Huang WX, Zhou X, Chen J and Li Z: Numb inhibits epithelial-mesenchymal transition via RBP-JK-dependent Notch1/PTEN/FAK signaling pathway in tongue cancer. BMC Cancer 19: 391, 2019.

14. Peng $\mathrm{H}$ and $\mathrm{Li} \mathrm{H}$ : The encouraging role of long noncoding RNA small nuclear RNA host gene 16 in epithelial-mesenchymal transition of bladder cancer via directly acting on miR-17-5p/metalloproteinases 3 axis. Mol Carcinog 2019 (Epub ahead of print).

15. Felipe Lima J, Nofech-Mozes S, Bayani J and Bartlett JM: EMT in breast carcinoma-a review. J Clin Med 5: E65, 2016.

16. Toh Y, Pencil SD and Nicolson GL: A novel candidate metastasis-associated gene, mta1, differentially expressed in highly metastatic mammary adenocarcinoma cell lines. cDNA cloning, expression, and protein analyses. J Biol Chem 269: 22958-22963, 1994.

17. Toh Y, Pencil SD and Nicolson GL: Analysis of the complete sequence of the novel metastasis-associated candidate gene, mta1, differentially expressed in mammary adenocarcinoma and breast cancer cell lines. Gene 159: 97-104, 1995.

18. Minard ME, Kim LS, Price JE and Gallick GE: The role of the guanine nucleotide exchange factor Tiam1 in cellular migration, invasion, adhesion and tumor progression. Breast Cancer Res Treat 84: 21-32, 2004.

19. Chen B, Ding Y, Liu F, Ruan J, Guan J, Huang J, Ye X, Wang S, Zhang G, Zhang X, et al: Tiam1, overexpressed in most malignancies, is a novel tumor biomarker. Mol Med Rep 5: 48-53, 2012

20. Yu LN, Zhang QL, Li X, Hua X, Cui YM, Zhang NJ, Liao WT and Ding YQ: Tiam1 transgenic mice display increased tumor invasive and metastatic potential of colorectal cancer after 1,2-dimethylhydrazine treatment. PLoS One 8: e73077, 2013.

21. Ferrara N, Gerber HP and LeCouter J: The biology of VEGF and its receptors. Nat Med 9: 669-676, 2003.

22. Kessenbrock K, Plaks V and Werb Z: Matrix metalloproteinases: Regulators of the tumor microenvironment. Cell 141: 52-67,2010.

23. Stetler-Stevenson WG: Matrix metalloproteinases in angiogenesis: A moving target for therapeutic intervention. J Clin Invest 103: 1237-1241, 1999.
24. Bourboulia D and Stetler-Stevenson WG: Matrix metalloproteinases (MMPs) and tissue inhibitors of metalloproteinases (TIMPs): Positive and negative regulators in tumor cell adhesion. Semin Cancer Biol 20: 161-168, 2010.

25. Gomes E and Rockwell P: p38 MAPK as a negative regulator of VEGF/VEGFR2 signaling pathway in serum deprived human SK-N-SH neuroblastoma cells. Neurosci Lett 431: 95-100, 2008.

26. Wang L, Liu T, Nishioka M, Aguirre RL, Win SS and Okada N: Activation of ERK1/2 and cyclin D1 expression in oral tongue squamous cell carcinomas: Relationship between clinicopathological appearances and cell proliferation. Oral Oncol 42: 625-631, 2006.

27. Li Q and Yang Z: Expression of phospho-ERK1/2 and PI3-K in benign and malignant gallbladder lesions and its clinical and pathological correlations. J Exp Clin Cancer Res 28: 65, 2009.

28. Yang Y, Park H, Yang Y, Kim TS, Bang SI and Cho D: Enhancement of cell migration by corticotropin-releasing hormone through ERK1/2 pathway in murine melanoma cell line, B16F10. Exp Dermatol 16: 22-27, 2007.

29. Henry C, Llamosas E, Knipprath-Meszaros A, Schoetzau A, Obermann E, Fuenfschilling M, Caduff R, Fink D, Hacker N, Ward R, et al: Targeting the ROR1 and ROR2 receptors in epithelial ovarian cancer inhibits cell migration and invasion. Oncotarget 6: 40310-40326, 2015.

30. Arocho A, Chen B, Ladanyi M and Pan Q: Validation of the 2-DeltaDeltaCt calculation as an alternate method of data analysis for quantitative PCR of BCR-ABL P210 transcripts. Diagn Mol Pathol 15: 56-61, 2006.

31. Jayachandran A, Dhungel B and Steel JC: Epithelial-to-mesenchymal plasticity of cancer stem cells: Therapeutic targets in hepatocellular carcinoma. J Hematol Oncol 9: 74, 2016.

32. Wang H, Wu Q, Zhang Y, Zhang HN, Wang YB and Wang W: TGF- $\beta 1$-induced epithelial-mesenchymal transition in lung cancer cells involves upregulation of miR-9 and downregulation of its target, E-cadherin. Cell Mol Biol Lett 22: 22, 2017.

33. Zhu G, Zhang Y, Wang Q, Che S, Yang Y, Chen L and Lin Z: The prognostic value of Tiam1 correlates with its roles in epithelial-mesenchymal transition progression and angiogenesis in lung adenocarcinoma. Cancer Manag Res 11: 1741-1752, 2019.

34. Liu CY, Lin HH, Tang MJ and Wang YK: Vimentin contributes to epithelial-mesenchymal transition cancer cell mechanics by mediating cytoskeletal organization and focal adhesion maturation. Oncotarget 6: 15966-15983, 2015.

35. Lin X, Zheng L, Song H, Xiao J, Pan B, Chen H, Jin X and Yu H: Effects of microRNA-183 on epithelial-mesenchymal transition, proliferation, migration, invasion and apoptosis in human pancreatic cancer SW1900 cells by targeting MTA1. Exp Mol Pathol 102: 522-532, 2017.

36. Roskoski R Jr: Small molecule inhibitors targeting the EGFR/ErbB family of protein-tyrosine kinases in human cancers. Pharmacol Res 139: 395-411, 2019.

37. Ding D, Huang H, Jiang W, Yu W, Zhu H, Liu J, Saiyin H, Wu J, Huang H, Jiang S and Yu L: Reticulocalbin-2 enhances hepatocellular carcinoma proliferation via modulating the EGFR-ERK pathway. Oncogene 36: 6691-6700, 2017.

38. Ye QH, Zhu WW, Zhang JB, Qin Y, Lu M, Lin GL, Guo L, Zhang B, Lin ZH, Roessler S, et al: GOLM1 modulates EGFR/RTK cell-surface recycling to drive hepatocellular carcinoma metastasis. Cancer Cell 30: 444-458, 2016.

39. Scaltriti M and Baselga J: The epidermal growth factor receptor pathway: A model for targeted therapy. Clin Cancer Res 12: 5268-5272, 2006.

40. Ikeda S, Tsigelny IF, Skjevik Å, Kono Y, Mendler M, Kuo A, Sicklick JK, Heestand G, Banks KC, Talasaz A, et al: Next-generation sequencing of circulating tumor DNA reveals frequent alterations in advanced hepatocellular carcinoma. Oncologist 23: 586-593, 2018.

41. Li R, Yanjiao G, Wubin H, Yue W, Jianhua H, Huachuan Z, Rongjian S and Zhidong L: Secreted GRP78 activates EGFRSRC-STAT3 signaling and confers the resistance to sorafeinib in HCC cells. Oncotargt 8: 19354-19364, 2017.

42. Panvichian R, Tantiwetrueangdet A, Sornmayura $P$ and Leelaudomlipi S: Missense mutations in exons 18-24 of EGFR in hepatocellular carcinoma tissues. Biomed Res Int 2015: 171845, 2015.

43. Chen BH and Giudice LC: Dysfunctional uterine bleeding. West J Med 169: 280-284, 1998. 
44. Solca F, Dahl G, Zoephel A, Bader G, Sanderson M, Klein C, Kraemer O, Himmelsbach F, Haaksma E and Adolf GR: Target binding properties and cellular activity of afatinib (BIBW 2992), an irreversible ErbB family blocker. J Pharmacol Exp Ther 343: 342-350, 2012.

45. Tu Y, Wang C, Yang Z, Zhao B, Lai L, Yang Q, Zheng P and $\mathrm{Zhu} \mathrm{W}$ : Discovery of novel quinazoline derivatives bearing semicarbazone moiety as potent EGFR kinase inhibitors. Comput Struct Biotechnol J 16: 462-478, 2018

46. Roof AK and Gutierrez-Hartmann A: Consider the context: Ras/ERK and PI3K/AKT/mTOR signaling outcomes are pituitary cell type-specific. Mol Cell Endocrinol 463: 87-96, 2018.

47. Fang ZT, Wang GZ, Zhang W, Qu XD, Liu R, Qian S, Zhu L, Zhou B and Wang JH: Transcatheter arterial embolization promotes liver tumor metastasis by increasing the population of circulating tumor cells. OncoTargets Ther 6: 1563-1572, 2013.

48. Ishiyama N, Lee SH, Liu S, Li GY, Smith MJ, Reichardt LF and Ikura M: Dynamic and static interactions between p120 catenin and E-cadherin regulate the stability of cell-cell adhesion. Cell 141: 117-128, 2010

49. Jiang WG, Grimshaw D, Martin TA, Davies G, Parr C, Watkins G, Lane J, Abounader R, Laterra J and Mansel RE: Reduction of stromal fibroblast-induced mammary tumor growth, by retroviral ribozyme transgenes to hepatocyte growth factor/scatter factor and its receptor, c-MET. Clin Cancer Res 9: 4274-4281, 2003.

50. Kalluri R and Weinberg RA: The basics of epithelial-mesenchymal transition. J Clin Invest 119: 1420-1428, 2009.

51. Puisieux A: Role of epithelial-mesenchymal transition in tumor progression. Bull Acad Natl Med 193: 2017-2032; discussion 2032-2034, 2009 (In French).

52. Wang ZC, Gao Q, Shi JY, Guo WJ, Yang LX, Liu XY, Liu LZ, Ma LJ, Duan M, Zhao YJ, et al: Protein tyrosine phosphatase receptor $\mathrm{S}$ acts as a metastatic suppressor in hepatocellular carcinoma by control of epithermal growth factor receptor-induced epithelial-mesenchymal transition. Hepatology 62: 1201-1214, 2015

53. Deng L, Tang J, Yang H, Cheng C, Lu S, Jiang R and Sun B: MTA1 modulated by miR-30e contributes to epithelial-to-mesenchymal transition in hepatocellular carcinoma through an ErbB2-dependent pathway. Oncogene 36: 3976-3985, 2017

54. Hashida S, Yamamoto H, Shien K, Miyoshi Y, Ohtsuka T, Suzawa K, Watanabe M, Maki Y, Soh J, Asano H, et al: Acquisition of cancer stem cell-like properties in non-small cell lung cancer with acquired resistance to afatinib. Cancer Sci 106 $1377-1384,2015$

55. Kang X, Lu P, Cui Y, Wang Y, Zhang Q, Gong Y and Xu Z: Bufalin reverses hepatocyte growth factor-induced resistance to afatinib in H1975 lung cancer cells. Zhonghua Zhong Liu Za Zhi 37: 490-496, 2015 (In Chinese)
56. Song L, Li W, Zhang H, Liao W, Dai T, Yu C, Ding X, Zhang L and Li J: Over-expression of AEG-1 significantly associates with tumour aggressiveness and poor prognosis in human non-small cell lung cancer. J Pathol 219: 317-326, 2009.

57. Jain RK, Duda DG, Clark JW and Loeffler JS: Lessons from phase III clinical trials on anti-VEGF therapy for cancer. Nat Clin Pract Oncol 3: 24-40, 2006.

58. Ng EW, Shima DT, Calias P, Cunningham ET Jr, Guyer DR and Adamis AP: Pegaptanib, a targeted anti-VEGF aptamer for ocular vascular disease. Nat Rev Drug Discov 5: 123-132, 2006.

59. Mukhopadhyay D, Nagy JA, Manseau EJ and Dvorak HF: Vascular permeability factor/vascular endothelial growth factor-mediated signaling in mouse mesentery vascular endothelium. Cancer Res 58: 1278-1284, 1998.

60. Zhang HH, Zhang Y, Cheng YN, Gong FL, Cao ZQ, Yu LG and Guo XL: Metformin incombination with curcumin inhibits the growth, metastasis, and angiogenesis of hepatocellular carcinoma in vitro and in vivo. Mol Carcinog 57: 44-56, 2018.

61. Brown DM and Regillo CD: Anti-VEGF agents in the treatment of neovascular age-related macular degeneration: Applying clinical trial results to the treatment of everyday patients. Am J Ophthalmol 144: 627-637, 2007.

62. Huang M, Huang B, Li G and Zeng S: Apatinib affect VEGF-mediated cell proliferation, migration, invasion via blocking VEGFR2/RAF/MEK/ERK and PI3K/AKT pathways in cholangiocarcinoma cell. BMC Gastroenterol 18: 169, 2018.

63. Fearnley GW, Smith GA, Abdul-Zani I, Yuldasheva N, Mughal NA, Homer-Vanniasinkam S, Kearney MT, Zachary IC, Tomlinson DC, Harrison MA, et al: VEGF-A isoforms program differential VEGFR2 signal transduction, trafficking and proteolysis. Biol Open 5: 571-583, 2016.

64. Almalki SG and Agrawal DK: ERK signaling is required for VEGF-A/VEGFR2-induced differentiation of porcine adipose-derived mesenchymal stem cells into endothelial cells. Stem Cell Res Ther 8: 113, 2017

65. Mu X, Zhao T, Xu C, Shi W, Geng B, Shen J, Zhang C, Pan J, Yang J, Hu S, et al: Oncometabolite succinate promotes angiogenesis by upregulating VEGF expression through GPR91-mediated STAT3 and ERK activation. Oncotarget 8: 13174-13185, 2017.

This work is licensed under a Creative Commons Attribution-NonCommercial-NoDerivatives 4.0 International (CC BY-NC-ND 4.0) License. 